\title{
VALOR NUTRICIONAL DE LA ALGARROBA (Prosopis pallida) EN LA ALIMENTACIÓNDEL CABALLO
}

Fernando Peñaloza A. ${ }^{1}$, Felipe San M artín H. ${ }^{2}$ y M iguel Ara G. ${ }^{3}$

\section{Abstract}

Thepresent research was carried out to estimatethenutritional value of carob bean (Prosopis pallida) in horses. Four rations with increasing carob bean levels (0; 20: 46.7 y $66.7 \%$ ) in abase-diet of barley and lucemehay wereeval uated. Four Anglo-Argentinien crossbred geldings were fed the four rations using a simple overchange model with an arrangement of the $4 \times 4$ Latin Square $A$ feeding-test consisting of lucemehay al onewas previously done, aiming at obtaining di gestibility of carob bean by differencein percentage with the treatment containing $66.77 \%$ carob bean. Digestibility of both dry and organic matter was $62.1 \%$ and $61.6 \%$, respectively. Theincrease of carob bean's content in rations reduced digestibility in most nutritional fractions of feeding rations, with theexception of ethereal extract Reductionfollowed alinear, significantpattem $(\mathrm{P} \varangle 0.05)$ for organic matter, nitrogen-freeextract, neutral detergent fibreand acid detergent fibre.

K ey words: carob bean, Prosopis pallida, digestibility, horse

\section{Resumen}

El presenteestudio sellevóa cabo conel objetivo deestimar el valor nutritivo dela al garroba (Prosopis pall lida) en cabal los. Se eval uaron cuatro raciones con niveles crecientes $(0 ; 20 ; 46.7$ y $66.7 \%)$ deal garroba, en una dieta base decebaday heno dealfal fa. Se usaron cuatro cabal l os castrados de cruce Anglo-Argentino, que fueron sometidos a las cuatro raciones, en un diseño de sobrecambio simple con arreglo deCuadrado Latino $4 \times 4$. Previamentesellevó a cabo un ensayo dealimentación con sól o heno deal falfa con la final idad de obtener la di gestibilidad de la al garroba sol a por diferencia con el tratamiento de $66.7 \%$ deal garroba. Ladigesti bil lidad dela materia seca y materiaorgánica fue de 62.1 y $61.6 \%$ respectivamente. El incremento de algarroba en la ración redujo la digestibilidad de la mayor parte de las fracciones nutricional es delas raciones, a excepción del extracto etéreo. El patrón de estareducciónfuelineal, significativo $(\mathrm{P} \varangle 0.05)$ para materia orgánica, extracto libre de nitrógeno, fibra detergente neutra y fibra detergente ácida.

Palabras clave: algarroba, Prosopis pallida, digestibilidad, caballo

1 Graduado dela Unidad de Postgrado, FMV-UNMSM

2 Laboratorio de Nutrición, FMV-UNMSM. E-mail: decanovet@ unmsmedu.pe

${ }^{3}$ Departamento de Producción Animal, FMV-UNMSM 


\section{NTRODUCCIÓN}

En los pasados 20 años se reunió suficienteinformación para permitir la formulación científica de raciones para cabal los que cubran sus requerimientos nutritivos. Las pruebas deal imentación han sido el principal método empl eado para determinar los requerimientos cual itativosy cuantitativos delamayoría de los nutrientes conocidos, así como para determinar el valor de los alimentos y las combinaciones dietéticas para el crecimiento, mantenimiento, trabajo, lactancia y reproducción.

En nuestro país la alimentación deca ball los en crianza intensiva es a base de grano de cebada y heno deal falfa; sin embargo, en unidades de Caballería del Ejército una parte de la dieta general mentees conformada por el fruto de algarrobo (Prosopis pallida), debido asual tadisponi bilidady bajo precio.

No existen trabajos realizados en nuestro medio sobreel uso deal garroba en la al imentación de cabal los. Sin embargo en países desarrollados se ha logrado establecer cierta relación entre los componentes químicos y los val ores de digestibilidad de los al imentos. Sehademostrado queladigestibilidad de todos los componentes del forraje disminuye con el aumento de la fibra cruda y la materia seca y se ha observado que el contenido de fibra cruda fueel mejor predictor de todas las digestibilidades (A plegate y Hershberger, 1969; Vander Nooty Gilbreath, 1970). Por otro lado, en estudios comparativos de alimentación entre vacunos, ovinos, conejos y caball os no se observaron diferencias significativas al emplear al imentos con menos del 15\% de fibra cruda (Hintz, 1969; Hintz etal., 1971; Sladey Hintz, 1969) por lo que se hace necesario eval uar directamente la digestibilidad de raciones conteniendo algarroba, componente que usual mente presenta val ores superiores de $15 \%$ defibracnuda. En base a lo antes expuesto, este trabajo tiene como objetivos estimar en cabal los $\mathrm{e}$ valor nutritivo de la algarroba y su digestibilidad en función de su inclusión en niveles crecientes en raciones a base de cebada y heno de alfalfa.

\section{Materiales y Métodos}

Ubicación

El estudio se realizó entre febrero y marzo de1994 en las instalaciones del Regimiento de Cabal lería "Escol ta" del Ejército Peruano, ubicado en el distrito del Agustino Provincia de Lima, a una altitud de $197 \mathrm{~m}$, latitud sur de $12^{\circ} 03^{\prime} 04^{\prime \prime}$, y a una longitud de 7659' 54" (INEI, 1997). Los análisis serea lizaronenel Laboratorio deBioquímica, Nur tricióny AlimentaciónA nimal delaFacultad de Medicina Veterinaria de la Universidad Nacional Mayor de San Marcos en Lima.

A nimales

Sesel eccionaron 4 cabal los castrados de 5 a 8 años de edad, con un peso promedio de $400 \mathrm{~kg}$, entreuna población de 130 cabaIlos cruzadosAnglo-Argentino. Antes deiniciar el experimento sedosificóalos animales con fenbendazol al $10 \%$ auna dosis de $1 \mathrm{ml} /$ $10 \mathrm{~kg}$ p.v., se les limó los dientes y fueron confinados en cabal lerizasindividuales. En la puerta de cada corral se indicó la ración que debía recibir el ani mal para cada período.

Tratamientos, alimentación y manejo de los animales

Cada uno de los animal es fue sometido a una al imentación secuencial con cuatro raciones con nivel es crecientes de al garroba $(0,20.0,46.7$ y $66.7 \%)$ y decrecientes dece bada $(66.7,46.7,20.0$ y $0 \%)$ y una cantidad constante de heno de alfalfa (33.3\%) (Cuadro 1). Cada período dela secuencia duró 12 días, con 7 días deacostumbramiento y 5 días derecolección deheces. Previamenteserear lizó un ensayo, al imentando a todos los animales con la dieta basal ( $5 \mathrm{~kg}$ de heno de 
Cuadro 1. Raciones usadas en la alimentación secuencial en el experimento [( $\mathrm{Kg} \mathrm{( \% )]}$

\begin{tabular}{lcccc}
\hline \multirow{2}{*}{ Insumos } & \multicolumn{5}{c}{ Tratamientos } \\
\cline { 2 - 5 } & $\mathrm{A}$ & $\mathrm{B}$ & $\mathrm{C}$ & $\mathrm{D}$ \\
\hline Algarroba & $5.0(66.7)$ & $3.5(46.7)$ & $1.5(20.0)$ & $0.0(00.0)$ \\
Cebada & $0.0(00.0)$ & $1.5(20.0)$ & $3.5(46.7)$ & $5.0(66.7)$ \\
Heno dealfalfa & $2.5(33.3)$ & $2.5(33.3)$ & $2.5(33.3)$ & $2.5(33.3)$ \\
\hline Total & $7.5(100.0)$ & $7.5(100.0)$ & $7.5(100.0)$ & $7.5(100.0)$ \\
\hline
\end{tabular}

alfalfa) por 7 días de acostumbramiento y 5 días de recolección de heces, con la finalidad de estimarse por diferencia, con los re sultados dela ración de $66.7 \%$ deal garroba, el coeficiente dedigestibilidad dela al garrobasola. Laalimentaciónfuecontrolada para mejorar lautilizacióndel régimeny sobretodo para evitar los diversos trastornos gastrointestinales a los que el caballo es especial mente sensible Por ello se consideró una cantidad igual de heno de alfalfa en todos los tratamientos. El horario de abrevado fuecada dos horas, a partir delas 06:00 hasta las 20:00 horas.

Las raciones se prepararon y pesaron el día anterior, de acuerdo al tratamiento correspondiente para cada caballo y cada periodo. El heno deal fal fay las vainas dealgaroba se cortaron en trozos pequeños. Lace bada se remojó en agua por 3 horas antes de su consumo. La ración se suministró en dos partes iguales, la primera a las 08:00 (3.75 kg) y la otra a las 14:00 horas (3.75 kg). En todo momento se registraron los consumos individuales delos caballos.

M uestras

Seobtuvieron muestras delosinsumos al inicio del ensayo para el análisis correspondiente.

Secolocaron bolsas col ectoras de he ces, fabricadas de lona y sujetadas con arneses a los caballos del estudio a partir de las 08:00 del primer día de la fase de reco- lección. El contenido de las bolsas fue evacuado a las 18:00 y 08:00 de cada día experimental. El contenido fuepesado y registrado, y seobtuvieron sub-muestras homogéneas en bol sas plásticas debidamenterotul adas y herméticamente cerradas. Las sub-muestras fueron al macenadas en congelación a-25ㅇ C para su posterior análisis. Las sub-muestras delas 18:00 horas deundíay delas 08:00 del día siguientesecombinaron paraformar una sol a sub-muestra, llegando a obtenersecinco sub-muestras por animal dentro de cada tratamiento.

\section{A nálisis de laboratorio}

Los al imentos componentes de las raciones, así como las muestras de heces fueron sometidas al análisis proximal (AOAC, 1990) estimándose materia seca, materia orgánica, proténa cruda, fibra cruda, extracto etéreo y extracto libre de nitrógeno. Adicional menteseestimó fibradetergenteneur tra y fibra detergente ácida (AOAC, 1990).

Determinación de la digestibilidad aparente

Los coeficientes de digestibilidad aparente in vivo de las fracciones del análisis proximal y deVan Soestseobtuvieron mediantelasiguientefórmula(VanSoest, 1982):

Coeficientededigestibilidad $(\%)=$

nutrienteingerido-nutrienteen heces $\times 100$ nutriente ingerido 
El ensayo previo con sólo heno dealfalfa sirvió para calcular el coeficiente de digestibilidad de la al garroba por diferencia (Lloyd et al., 1987) con los resul tados obtenidos con la ración del $66.7 \%$ de al garroba.

En base a los resultados del val or nutritivo y coeficientes de digestibilidad delos insumosy las raciones seevaluólacalidad de acuerdo al os valoressiguientes: bajo (<40\%), media (41-70\%) y alta (>71\%).

Diseño experimental

Se utilizó un diseño de sobrecambio simplecon arreglo de Cuadrado Latino $4 \times 4$. Los parámetros evaluados fueron someti dos a análisis de varianza, y en caso de hallar diferencias significativas entre tratamientos, estas fueron sometidas a la prueba de com- paración de medias por el método de Dife rencia MínimaSignificante(DMS) protegida (Steel y Torrie, 1987). Laestimación del efecto de los niveles de al garroba en una dieta base de cebada y heno de alfalfa sobre la digestibilidad de las fracciones se efectuó mediante análisis de regresión lineal y cuadrática.

\section{RESULTADOS Y DiscusióN}

Los resultados del análisisproximal de las raciones, de sus componentes y del fraccionamiento delafibra (Cuadro 2) muestran que las mayores diferencias se observan en el contenido defibra cruda delas raciones, el cual se incrementa de 13.6 a $23.8 \%$ a medida queel nivel deal garroba se incrementa.

Cuadro 2. Análisis proximal y de Van Soest de las raciones utilizadas y de sus componentes

\begin{tabular}{lrrrrrrr}
\hline \multirow{2}{*}{ Fracción } & \multicolumn{3}{c}{ Raciones (\% dealgarroba) } & \multicolumn{3}{c}{ Componentes } \\
\cline { 2 - 8 } & 66.7 & 46.7 & 20.0 & 0 & Algarroba & Cebada & Heno alfalfa \\
\hline MS & 87.5 & 87.2 & 86.8 & 86.4 & 87.9 & 86.3 & 86.7 \\
Cenizas & 6.1 & 5.9 & 5.7 & 5.5 & 4.0 & 3.1 & 10.3 \\
MO & 93.9 & 94.1 & 94.3 & 94.5 & 96.0 & 96.9 & 89.7 \\
PC & 12.2 & 12.3 & 12.4 & 12.5 & 9.0 & 9.3 & 18.8 \\
FC & 23.8 & 20.8 & 16.7 & 13.6 & 19.7 & 4.3 & 32.2 \\
EE & 1.7 & 1.6 & 1.6 & 1.6 & 1.8 & 1.8 & 1.4 \\
ELN & 56.2 & 59.4 & 63.6 & 66.8 & 65.5 & 81.5 & 37.3 \\
FDN & 53.9 & 54.4 & 55.2 & 55.7 & 46.7 & 49.3 & 68.5 \\
FDA & 48.7 & 49.2 & 49.8 & 50.4 & 41.5 & 43.9 & 63.3 \\
\hline
\end{tabular}

$M S=$ materia seca, $M O=$ materia orgánica, $P C=$ proteína cruda, $F C=$ fibra cruda, EE =extracto etéreo,

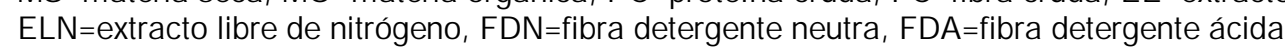


Coeficiente de digestibilidad aparente de la algarroba

Los coeficientes dedigestibilidad dela algarroba, obtenidos por diferencia con los resultados dela digestibilidad del tratamiento previo con heno de al fal fa se muestran en $\mathrm{e}$ Cuadro 3. Estos val ores son inferiores a los obtenidos por Coronado (1950) en ovinos y particularmente los val ores de fibra cruda.

\begin{tabular}{cc} 
Cuadro 3. & \multicolumn{2}{c}{$\begin{array}{l}\text { Coeficientes de digestibilidad } \\
\text { aparente de las fracciones } \\
\text { del análisis proximal y de } \\
\text { Van Soest de la al garroba en } \\
\text { caballos }\end{array}$} \\
\hline & \\
\hline Fracción & Coeficiente de \\
& digestibilidad (\%) \\
\hline MS & 62.1 \\
MO & 61.6 \\
PC & 61.5 \\
FC & 6.9 \\
EE & 20.9 \\
ELN & 78.6 \\
FDN & 25.6 \\
FDA & 23.3
\end{tabular}

MS = materia seca, MO = materia orgánica, $\mathrm{PC}=$ proteína cruda, $\mathrm{FC}=$ fibra cruda, $\mathrm{EE}=$ extracto etéreo, ELN = extracto libre de nitrógeno, FDN = fibra detergente neutra, FDA = fibra detergente ácida

Es probablequeesta diferencia pue da ser debida a una mejor digestión de la fibra por los ovinos, por su condición de poligástricos, debido a que la degradación microbiana del os carbohidratos complejos es mayor en el rumen del vacuno queen el ciego del caballo; y la mayor degradación microbianaestéen relación a una mayor población y calidad microbiana en el rumen (Maynard et al., 1986).

En cuanto a los resultados por animal, seobservó diferencias marcadas entre caball los paraladigestibilidad delafibracrut da. Deacuerdo a los resultados obteni dos se puede indicar que la al garroba en I a alimentación del caballoes debajadigestibilidad para fibra cruda, extracto etéreo, fibra detergente neutra y fibra detergente ácida; de digestibilidad media para materia seca, ma teria orgánica y proteína cruda; y, de di gestibili dad al ta para extracto libre denitrógeno, debido a quelafracción mayoritaria de la pul pa de la al garroba está constitui da por sacarosa (95.4\%) y el resto por glucosa, fructuosa, gal actosa, xilosa, arabinosa y fucosa (Grados et al.,1994).

Los resultados de los análisis de varianza indican que la inclusión de niveles crecientes de al garroba en una dieta base de cebada y heno de alfalfa afectaron la digestibilidad de la materia seca, materia orgánica, extracto libre denitrógeno ( $\mathrm{P} \varangle 0.01$ ), fibra detergente neutra $(P \varangle 0.05)$ y fibra de tergenteácida $(P \varangle 0.01)$, peronolaproténacnuda, fibra cruda y extracto etéreo (Cuadro 4).

El análisis demedias (DMS) serealizó con aquellas fracciones con significancias estadísticas, encontrándosequeel coeficiente de digesti bilidad disminuye a medida que seincrementan los niveles deal barroba en la ración ( $P \varangle 0.05$, Cuadro 5). Sin embargo, $y$ dependiendo de la fracción analizada, al gunas raciones no fueron diferentes $(P>0.05)$.

El análisis de regresión lineal mostró diferencias significativas entre raciones ( $\mathrm{P} \varangle 0.05$, Cuadro 6) para materia orgánica, extracto libre de nitrógeno, fibra detergente neutra y fibra detergente ácida.

Este análisis se ilustra en los gráficos de la Fig. 1 donde se observa que a excepción del extracto etéreo, los niveles crecientes deal garroba redujeron en formal ineal la digestibilidad detodas las fracciones. Latendencia en reducción de la digestibilidad de las fracciones es explicablefundamental mente en términos del contenido de fibra cruda de los tratamientos. Estos resultados están de acuerdo con los reportados por A plegate 
Cuadro 4. Cuadrados medios del análisis de varianza para las diferentes fuentes de variabilidad y para los componentes de análisis proximal y de Van Soest de raciones con niveles crecientes de al garroba en la al imentación de caballos

\begin{tabular}{lccccccccc}
\hline \multirow{2}{*}{ Fuente } & GL & \multicolumn{8}{c}{ Cuadrados medios } \\
\cline { 3 - 10 } & & MS & MO & PC & FC & EE & ELN & FDN & FDA \\
\hline Periodos & 3 & 3.51 & 2.0 & 28.77 & 13.38 & 52.88 & 3.50 & 7.01 & 12.29 \\
Caballos & 3 & 0.70 & 0.42 & 21.43 & 2.21 & 63.46 & 0.54 & 19.26 & 7.69 \\
$\begin{array}{l}\text { Niveles de } \\
\text { algarroba }\end{array}$ & 3 & $115.41^{* *}$ & $140.08^{* *}$ & $42.75^{\text {ns }}$ & $60.66^{\text {s }}$ & $201.98^{\text {ns }}$ & $67.72^{* *}$ & $341.35^{*}$ & $484.03^{* *}$ \\
Error & 6 & 10.95 & 9.57 & 10.62 & 31.04 & 57.03 & 5.45 & 44.45 & 25.20 \\
\hline
\end{tabular}

$\mathrm{MS}=$ materia seca, $\mathrm{MO}=$ materia orgánica, $\mathrm{PC}=$ proteína cruda, $\mathrm{FC}=$ fibra cruda, $\mathrm{EE}=$ extracto etéreo, $E L N=$ extracto libre de nitrógeno, FDN = fibra detergente neutra, FDA = fibra detergente ácida ** $=\mathrm{P}>0.01, *=\mathrm{P}>0.05, \mathrm{~ns}=$ no significativo

Cuadro 5. Análisis de medias de la digestibilidad aparente de raciones con niveles crecientes de al garroba en la alimentación de caballos

\begin{tabular}{ccccc}
\hline \multirow{2}{*}{ Fracción } & \multicolumn{4}{c}{ Raciones (\% algarroba) } \\
\cline { 2 - 5 } & 66.7 & 46.7 & 20.0 & 0 \\
\hline MS & $57.9^{\mathrm{a}}$ & $59.7^{\mathrm{a}}$ & $62.2^{\mathrm{a}}$ & $70.1^{\mathrm{b}}$ \\
MO & $58.2^{\mathrm{a}}$ & $60.4^{\mathrm{ab}}$ & $63.7^{\mathrm{b}}$ & $71.7^{\mathrm{c}}$ \\
ELN & $73.3^{\mathrm{a}}$ & $75.3^{\mathrm{a}}$ & $77.1^{\mathrm{a}}$ & $82.9^{\mathrm{b}}$ \\
FDN & $30.1^{\mathrm{a}}$ & $36.6^{\mathrm{a}}$ & $39.9^{\mathrm{a}}$ & $52.1^{\mathrm{b}}$ \\
FDA & $31.3^{\mathrm{a}}$ & $35.9^{\mathrm{ab}}$ & $42.8^{\mathrm{b}}$ & $56.6^{\mathrm{c}}$
\end{tabular}

MS = materia seca, $M O=$ materia orgánica, $E L N=$ extracto libre de nitrógeno, FDN = fibra detergente neutra, FDA = fibra detergente ácida

* Letras diferentes entre columnas indican diferencias significativas $(P<0.05)$ a la prueba de DMS protegida

y Hershberger (1969) y Vander Noot y Gilbreath (1970), quienes indican que la digestibilidad de todos los componentes del forrajedisminuyen con el aumento delafibra cruda en la materia seca.

El efecto cuadrático no fuesignificativo para ninguna de las fracciones. La predominancia de un efecto lineal y la ausen- cia de efecto cuadrático signi ficativo indican que el efecto dela inclusión de niveles cre cientes de algarroba en una dieta base de cebaday heno dealfalfasobreladigestibilidad de las fracciones del análisis proximal y de Van Soestes aditivo, por lo tanto existecierta confianza en la representatividad de los val ores dedigesti bili dad del a a garroba obtenidos por diferenciaa partir del ensayo previo. 


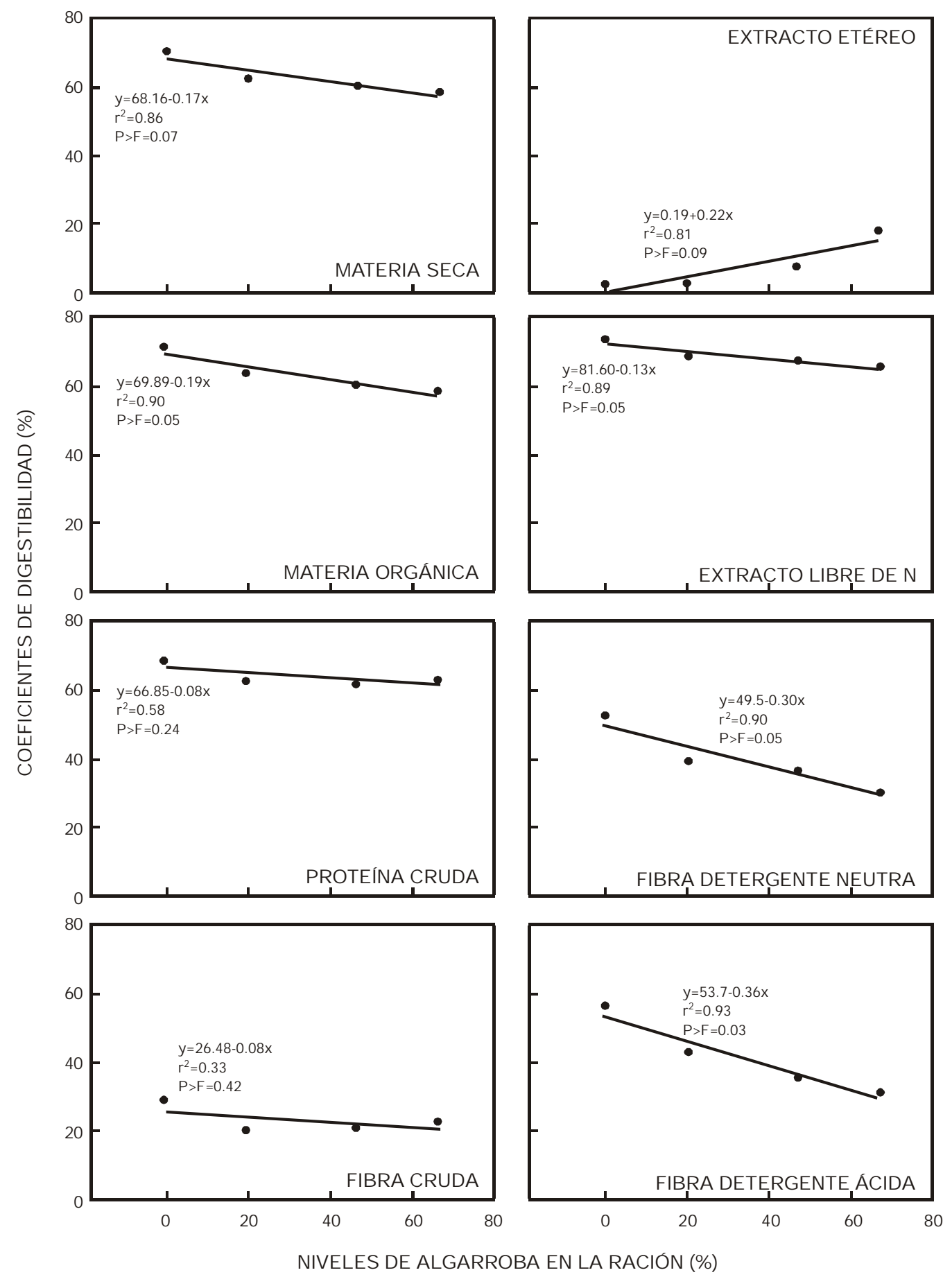

Fig. 1. Efecto de los niveles de algarroba sobre los coeficientes de digestibilidad parcial de las fracciones del análisis proximal y de van Soest 
Cuadro 6. Cuadrados medios de análisis de la regresión lineal para los diferentes componentes de análisis proximal y de Van Soest de raciones con niveles crecientes de al garroba en la alimentación de cabal los

\begin{tabular}{cccccccccc}
\hline \multirow{2}{*}{ Fuente } & \multirow{2}{*}{ GL } & \multicolumn{8}{c}{ Cuadrados medios } \\
\cline { 3 - 10 } & & MS & MO & PC & FC & EE & ELN & FDN & FDA \\
\hline Modelo & 1 & $75.10^{\text {ns }}$ & $94.66^{*}$ & $18.55^{\text {ns }}$ & $16.46^{\text {ns }}$ & $120.67^{\text {ns }}$ & $45.90^{*}$ & $230.37^{*}$ & $339.36^{*}$ \\
Error & 2 & 5.87 & 5.16 & 6.84 & 16.27 & 13.40 & 2.70 & 12.58 & 12.82 \\
\hline
\end{tabular}

$*=\mathrm{P}>0.05, \mathrm{~ns}=$ no significativo

\section{CONCLUSIONES}

1. Ladigestibilidad delaalgarroba paracaballosadul tos es de $62 \%$. Destacalabaja digestibilidad dela fibra cruda (6.9\%).

2. Laracióntradicional (sinincluir al garroba) esla demayor digestibilidad paratodaslas fraccionesnutricionales, a excepción del extracto etéreo.

3. El incremento de al garroba en la ración en cabal los reducel ineal mentel os coeficientes dedigestibilidad dela mayoría de las fracciones nutricionales.

4. Entérminos económicos, medido enfunción por kg demateria seca digerida, los menores precios se obtuvieron a mayoresinclusiones deal garrobaen la ración.

\section{Literatura Citada}

1. Association of Official Agricultural Chemists. 1990. Official methods of analysis. $15^{\text {th }}$ edition. Arlington.

2. Applegate, C.S.; T.V. Hershberger. 1969. Evaluation of in vitro and in vivo cecal fermentation tecniques for estimating the nutritive value of forages for equine. J. Anim Sci. 28: 18-22.

3. Coronado, J . 1950. Ladigestibilidad de las vainas deal garrobo enel ganado ovino. (Tesis) Escuela Nacional deAgricultura. Lima. $50 \mathrm{p}$.

4. Grados, N; L. Bravo; F. Saura-Calixto. 1994. Estudio comparativo entreal garroba peruana(Prosopispallida) y Mediterránea
(Ceratoniasiliqua). BoléńndelaSociedad Químicadel Perú60: 103-117.

5. Hint, H. 1969. Review article Equine nutrition comparisons of digestion coefficientobtained with cattle, sheep, rabbitsand horses. The Veterinarian 6: 45-51.

6. Hintz, H.F.; S.J . Roberts; S.W. Sabin; H.F. Schryver. 1971. Energy requeriments of lighthorses for various activities. J. Anim Sci. 32: 100-102.

8. Instituto Nacional de Estadística e I nformática. 1997. Guía INEI. Lima. $2^{\text {da }}$ ed., Edit. Continental de Comunica ciones S.A. Tomo I, $616 \mathrm{p}$.

9. Lloyd L.E.; B.E. McDonald; E.W. Crampton. 1987. Fundamentos de Nutrición. España EDAF, S.A. 464 p.

10. Maynard, L.A.; J.K. Loosli; H.F. Hintz; R.G. Warner. 1986. Nutrición animal. México. Editorial Mc Graw-Hill $7^{\mathrm{ma}}$ ed. $640 \mathrm{p}$.

11. Slade, L.M.; H.F. Hintz. 1969. Comparison of digestion in horses, ponies, rabbits, and guinea pigs. J. Anim Sci. 28: 842-843.

12. Steel, R.G.D.; J.H. Torrie, 1985. Bioestadística: Principiosy procedimientos. $2^{\text {da }}$ ed. Ed. McGraw-Hill Latinoamericana, S.A. 622 p.

13. Vander Noot, G.W.; E.C. Gilbreath. 1970. Comparativedigestibility of components of forage by gel dings and steers. J. Anim Sci. 33: 351-355.

14. Van Soest, P. 1982. Nutritional ecology of theruminant $O \& B$ Books Inc. 373p. 